\title{
Research on Weapon Target Allocation of Synthetic Detachment Considering Emergency Target
}

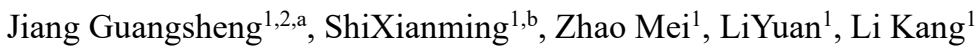 \\ ${ }^{1}$ Shijiazhuang Campus, AEU, Shijiazhuang, China \\ 2.Shijiazhuang Campus, Army Infantry College of PLA,Shijiazhuang, China
}

\begin{abstract}
In view of the problem of emergency target handling and reasonable attack in the combat process of synthetic detachment, this paper analyzes the main characteristics of emergency target, judges the threat degree of emergency target with "threat factor function", and controls the output of the solution by solving the objective function of "threat factor function", so as to ensure that the emergency target with great influence can be handled quickly. By improving the weapon target allocation model and using the solution control strategy, the simulation results show that this method can effectively improve the overall strike efficiency and the processing speed of emergency targets.
\end{abstract}

\section{Introduction}

WTA-Weapon Target Assignment was first proposed and applied in the field of air defense operations ${ }^{12}$. It is an important means to optimize firepower allocation and improve combat effectiveness, and it is also an important method to realize intelligent close cooperation among weapon platforms, and it is also an urgent problem to be solved to improve the intelligent decision-making level of combat command. The fundamental purpose of WTA problem is to distribute weapon platforms with different properties and uses to attack different targets through calculation and analysis, in order to obtain the maximum attack efficiency ${ }^{3}$. Literature ${ }^{4}$ puts forward the problem of dealing with new targets by solving utility function; in the literature ${ }^{56}$, the method of rapid fire distribution for emergency targets of synthetic detachment was put forward, which solved the problem of dealing with emergency targets to a certain extent, but none of the above methods specifically gave the discrimination and treatment methods of emergency targets. In this paper, combined with the operational characteristics of synthetic detachment, the concept of "threat factor function" is introduced, and a strike model considering the emergence of emergency targets and based on variable threats is proposed, which makes the model more suitable for the actual combat situation of synthetic detachment6. The improved ant colony algorithm is used to terminate the control and solve the model, thus ensuring that the emergency threat targets can be struck in time and effectively.

\section{BASIC ASSUMPTIONS AND PRINCIPLES OF WEAPON TARGET ALLOCATION OF SYNTHETIC DETACHMENT}

\subsection{Basic assumptions}

Synthetic detachment combat is a cooperative attack and defense battle of multi-weapon platforms. From the analysis of combat styles, it mainly includes three basic combat styles: offensive combat, defensive combat and encounter combat ${ }^{7}$. The process of offensive combat and defensive combat usually includes several combat stages, and the encounter situation is relatively complex, which mainly strikes the target according to the threat degree of the target. In order to facilitate the description and research of the problem, this paper takes the offensive combat of synthetic detachment as an example to explain and assume the basic principles in the combat process of synthetic detachment as follows:

The situation of targets in combat will change constantly with the combat process, and the appearing and disappearing targets should be added or reduced in time in the allocation model;

2.1.1 The status of weapon platform in combat is defined as two types: one is in distributable state (weapons and ammunition are ready and can be ordered to attack at any time), which is expressed by; second, it is in a nondistributable state (the weapon is in a state of adjustment and preparation such as shooting and ammunition loading), which is used to express;

2.1.2 During operation, the target may suddenly appear in a certain area by means of terrain, smoke screen and interference. Therefore, we should first judge whether the

\footnotetext{
aSilence12002@163.com bsxm@nudt.edu.cn
} 
target is an emergency target and start the emergency target strike plan;

2.1.3 The interference effects of command, interference, suppression and missile types are not considered in the combat process;

2.1.4 Synthetic detachments are mostly direct-aiming weapon platforms. When distributing weapon targets, they only carry out optimized attacks on targets within the attack range of this weapon platform;

2.1.5 In the combat of synthetic detachment, it is necessary to combine certain tactics, follow the principles of reducing the exposure of one's own targets, opening fire before the enemy, and starting damage ${ }^{8}$, and optimize the distribution of weapon targets on this basis.

\subsection{Basic principles}

Compared with ground air defense, air combat, naval warfare and other weapon target allocation, the weapon target allocation of synthetic detachment is more complicated due to the influence of timeliness, target quantity, situation change and tactical application, and the following principles should be followed in the allocation process.

2.2.1 Give priority to attacking urgent targets; when the system judges that there is an emergency target, it must immediately output the current optimal solution, and give priority to using weapons with high strike efficiency to attack the emergency target.

2.2.2 Follow the principle of discovery is destruction; synthetic detachments are mostly direct-aiming weapons, and the destruction of the target means that the target is destroyed. Therefore, in the process of fighting, we should minimize the exposure of our weapon platform on the basis of persisting in developing firepower.

2.2.3 Preferentially attack targets with high damage probability; under the same conditions, priority should be given to attacking targets with high damage probability.

\section{THREAT FACTOR FUNCTION OF EMERGENCY TARGET}

Synthetic teams fight, and there are many kinds of fihting weapons between the enemy and the enemy, the battlefield situation changes rapidly, and the target parameters change rapidly. With the advancement of the combat process, the targets temporarily added in specific combat areas often have unexpected detection and strike effects, and if the urgent targets are not handled in time, they will cause great losses. Therefore, the handling of urgent targets determines the development of the war situation. In synthetic detachment operations, the threat factors of emergency targets are related to the weapon effectiveness of emergency targets and the distance between weapon platform and targets.

\subsection{Weapon effectiveness}

According to the nature of different weapon platforms, weapon effectiveness has a fixed weapon effectiveness value, and different weapon effectiveness has different strike effectiveness against targets.

\subsection{Distance factor}

For emergency targets that suddenly appear in a specific area, the closer the distance, the greater the threat to our weapon platform, and the smaller the distance, the smaller the impact; for other targets such as reconnaissance, the influence factor of distance is relatively small, and it mainly has a great influence on the follow-up of combat.

$$
R_{j}=\left\{\begin{array}{cl}
1, & r \leq r^{\prime} \\
\left(\frac{r-r^{\prime \prime}}{r^{\prime}-r^{\prime \prime}}\right)^{2} & , r^{\prime}<r \leq r^{\prime \prime} \\
0, & r^{\prime \prime}<r
\end{array}\right.
$$

Distance factor, formula (2) reflects the influence of distance factor of target on threat factor. In the simulation calculation, the distance factor of the new target should be randomly generated in the uniform distribution.

Therefore, the threat factor function can be obtained:

$$
N_{j}=\alpha_{j} \cdot R_{j}=\alpha_{j} \cdot\left\{\begin{array}{cl}
1, & r \leq r^{\prime} \\
\left(\frac{r-r^{\prime \prime}}{r^{\prime}-r^{\prime \prime}}\right)^{2} & , r^{\prime}<r \leq r^{\prime \prime} \\
0, & r^{\prime \prime}<r
\end{array}\right.
$$

\section{ESTABLISHMENT OF WEAPON TARGET ALLOCATION MODEL OF SYNTHETIC DETACHMENT}

\subsection{General allocation model}

For the attacking party (Red Party) in the course of combat, the purpose of weapon target allocation is to expect to obtain the maximum damage effect, eliminate all kinds of enemy threat forces, and gradually reduce the remaining battlefield value of the defending party (Blue Party), so the enemy's remaining battlefield value is used for modeling. Red has a total of weapons, and when red weapons attack blue, they can only attack one blue target at a time. The blue side has a total of targets. The battlefield value of the target is expressed by, and the battlefield value of the target decreases with the continuous attack. Let the damage efficiency of the red weapon attacking the blue target be, where $i=1,2 \ldots x ; j=1,2 \ldots y$, let it represent the decision of the No. $i$ weapon platform to attack the No.j targets in Blue. The WTA model of synthetic detachment can be constructed as follows:

$$
\max U=\sum_{j=1}^{y}\left(u_{j} \cdot\left(1-\prod_{i=1}^{x}\left(1-p_{i j}\right)^{c^{i j}}\right)\right)
$$

Constraints are:

$$
\text { s.t. }\left\{\begin{aligned}
\sum_{j=1}^{y} c^{i j} \leq 1 \quad j=1,2,3 \cdots y \\
\sum_{i=1}^{x} c^{i j} \leq n \quad i=1,2,3 \cdots x \\
c^{i j} \in\{0,1\}
\end{aligned}\right.
$$


1) $\quad A=\left(c^{i j}\right)_{x \times y}, \quad c^{i j} \in\{0,1\}$ is weapon target allocation matrix. among them $c^{i j}=0$ show No. $i$ weapons don't strike No. $j$ target, $c^{i j}=1$ show No. $i$ weapons strike No. $j$ target.

2) Constraint condition (1) $c^{i j} \leq 1$, means that the same weapon platform can only strike at most one target at the same time.

3) Constraint condition (2) $c^{i j} \leq n$, indicates that the number of weapon platforms attacking the same target at the same time shall not be greater than $n$.

4) $\quad u_{j}$ is battlefield value of target, representing the battlefield importance of the j-th target.

According to the basic principles and characteristics of synthetic detachment operations, enemy targets may suddenly appear in a certain area by using various cover conditions. These targets may be attack targets such as helicopters and portable artillery, which pose a great threat to our side and must be dealt with urgently; targets may also be reconnaissance targets: reconnaissance units, unmanned aerial vehicles, observation equipment, etc., which are important targets and may have a great impact on the subsequent combat process.

Accord to that basic model of weapon target allocation, it is assumed that there are q weapon platform in red that can attack emergency targets, and the damage probabilities are $\left\{p_{1}, p_{2} \cdots p_{q}\right\}$.In the analysis of reference, the strike efficiency standard of weapon platform is determined, and the numerical value of the strike efficiency standard is determined by Delphi method as 0.7 , and its constraint conditions are as follows:

$$
\text { s.t. } 1-\prod_{i=1}^{q}\left(1-\mathrm{p}_{i j}\right) \leq 0.7 \quad j=1,2 \cdots y
$$

By increasing the number of weapon platforms, the standard of attack efficiency can be achieved within a certain attack time. Its drawback is that when a target with a high degree of threat appears, the target allocation system will attack the target by continuously concentrating more such firepower until the surplus value of the target reaches the prescribed standard. In actual combat operations, when some emergency targets appear, they must follow the principle that they are discovered and destroyed. Therefore, in the optimized attack area, we should find a weapon platform with appropriate attack distance and the highest damage efficiency, reduce unnecessary fire shooting, and achieve the effect of oneshot damage to the target, so as to meet the basic principles of fire attack of synthetic detachment and the processing procedures for emergency targets.

\subsection{Attack optimization function}

When attcking the target, the first enemy fire, the first hit, the delayed exposure, and the close-range shooting are the key factors that affect the attack efficiency in the course of combat. According to the stage division of the synthetic detachment's offensive combat, the attack methods required for attacking the enemy are different in different stages. Only by combining the firepower attack with the tactics of each combat stage can the attack efficiency be effectively improved. In the process of combat, the weapons of the synthetic detachment are mostly directaiming weapon platforms, and the attack distance and other factors directly determine the strike effect. Therefore, by introducing the attack optimization function, each weapon platform is optimized to strike at the best attack distance and the highest hit damage probability, so as to obtain better strike effect ${ }^{9}$, then:

$$
\begin{gathered}
G(\mathrm{x})=\left\{\begin{array}{cc}
0 & x<\alpha \\
\delta\left(\frac{x-\alpha}{\gamma-\alpha}\right)^{q_{1}} & \alpha \leq x<\gamma \\
1-(1-\delta)\left(\frac{x-\beta}{\gamma-\beta}\right)^{q_{2}} & \gamma \leq x<\beta \\
1 & \beta \leq x
\end{array}\right. \\
\mathrm{x} \in[0,1]
\end{gathered}
$$

The image is shown in Figure 1:

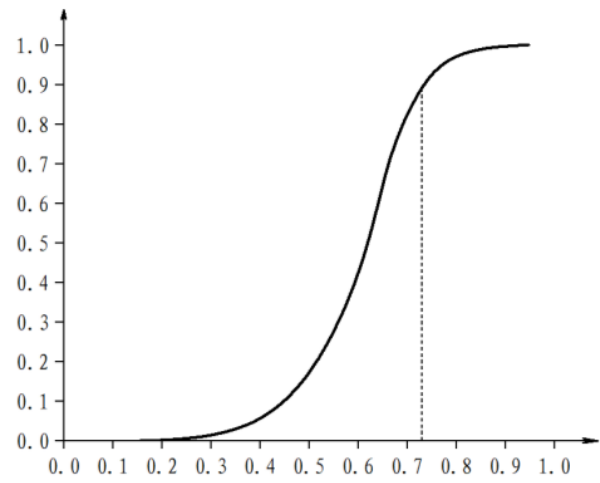

Fig1. Optimization function curve

By optimizing the function curve, it can be found that the slope is the largest when the function is between 0.65 and 0.75 , and the hit probability is the largest at this time. In order to ensure the striking effect, we choose to fire when the optimization function value is greater than 0.7 , at which time the hit damage probability is the highest. The addition of optimization function can not only improve the attack efficiency, but also effectively avoid the problems such as blind firing, low hit probability and target exposure.

At this point, the original allocation model is transformed into:

$$
\begin{aligned}
& \min U=\sum_{j=1}^{y} u_{\mathrm{j}} \cdot N_{u} \cdot G\left(\prod_{i=1}^{x}\left(1-p_{i j}\right)^{c^{i j}}\right) \\
& \text { s.t. }\left\{\begin{array}{l}
\sum_{j=1}^{y} c^{i j} \leq 1 \quad j=1,2,3 \cdots y \\
\sum_{i=1}^{x} c^{i j} \leq n \quad i=1,2,3 \cdots x \\
c^{i j} \in\{0,1\}
\end{array}\right.
\end{aligned}
$$

After the model is improved, it can effectively ensure that in the process of weapon target matching, as far as possible, a weapon platform with the same damage efficiency as the threat degree of the target is selected to attack the target. 


\subsection{Emergency target handling}

Set the threat factor threshold $W_{f}$, and judge whether the target is an urgent target by comparing the value of the threat factor function. Assuming that the a goal is an urgent goal, then

$$
\max _{j=1,2 \cdots x}\left\{N_{j a}\right\} \geq W_{f}
$$

That is : $\max _{j=1,2 \cdots x}\left\{\alpha_{j a} \times R_{j a}\right\} \geq W_{f}$

Here, the threat factor threshold is used to judge whether the target is an emergency target. Its advantage is that it can avoid the emergency attack on the target which appears urgently but has little threat, resulting in unnecessary exposure of its own target and waste of ammunition. Here, based on the commander's experience, we set the threat factor threshold as 0.72 . When the target threat factor value reaches more than 0.72 , start the emergency target strike program. Emergency targets are often threatening and have obvious influence on the combat process. Therefore, weapon platforms with high hit accuracy and high damage probability must be selected to attack emergency targets. Considering the costeffectiveness factors in the process of combat implementation, synthetic detachments usually use weapons with high strike efficiency and high value in a centralized and unified way, so as to play the role of winning by one strike in an emergency.

\subsection{Algorithm termination control}

According to the analysis of the content in the second part, combined with the operational characteristics of the synthetic detachment, the algorithm for solving the model must be based on time optimization ${ }^{10}$ and strike effect. In order to meet the fast output results of synthetic detachment and improve the strike efficiency to a certain extent, we adopt the following strategies to control the output results:

Strategy 1: The change value of strike effectiveness assigned by monitoring model for 15 times is less than 0.05

Strategy 2: If the threat factor value is greater than 0.72 , the operation is finished and the current optimal solution is output.

\section{SIMULATION EXAMPLE}

There are 10 weapon platforms $\mathrm{W}_{1} \sim \mathrm{W}_{10}$ in Red, which attack 5 targets $\quad M_{1} \sim M_{5}$ in Blue. A new target is randomly generated in the course of combat, and its threat factor is randomly generated, which obeys the uniform distribution of (0.7-0.8). The attack probability of weapon platform against each target is randomly generated, and the parameters obey the normal distribution of $(p, 0.3)$. The strike probability is shown in TABLE 1.
Table1. Probability of Weapon Attack on Target

\begin{tabular}{cccccc}
\hline & $\mathrm{M}_{1}$ & $\mathrm{M}_{2}$ & $\mathrm{M}_{3}$ & $\mathrm{M}_{4}$ & $\mathrm{M}_{5}$ \\
\hline $\mathrm{W}_{1}$ & 0.78 & 0.68 & 0.23 & 0.70 & 0.48 \\
$\mathrm{~W}_{2}$ & 0.76 & 0.47 & 0.25 & 0.64 & 0.34 \\
$\mathrm{~W}_{3}$ & 0.51 & 0.28 & 0.23 & 0.47 & 0.73 \\
$\mathrm{~W}_{4}$ & 0.56 & 0.29 & 0.80 & 0.35 & 0.77 \\
$\mathrm{~W}_{5}$ & 0.26 & 0.32 & 0.61 & 0.45 & 0.25 \\
$\mathrm{~W}_{6}$ & 0.34 & 0.64 & 0.26 & 0.59 & 0.27 \\
$\mathrm{~W}_{7}$ & 0.75 & 0.53 & 0.41 & 0.66 & 0.44 \\
$\mathrm{~W}_{8}$ & 0.29 & 0.79 & 0.26 & 0.20 & 0.47 \\
$\mathrm{~W}_{9}$ & 0.27 & 0.33 & 0.20 & 0.33 & 0.64 \\
$\mathrm{~W}_{10}$ & 0.23 & 0.64 & 0.33 & 0.21 & 0.76 \\
\hline
\end{tabular}

The battlefield value parameters of the target obey the normal distribution of $\left(u_{j}, 0.4\right)$, and the parameters are shown in TABLE 2.

Table2. Target battlefield value

\begin{tabular}{cccccc}
\hline Target & $\mathrm{M}_{1}$ & $\mathrm{M}_{2}$ & $\mathrm{M}_{3}$ & $\mathrm{M}_{4}$ & $\mathrm{M}_{5}$ \\
\hline $\begin{array}{c}\text { Strike } \\
\text { probability }\end{array}$ & 0.25 & 0.82 & 0.69 & 0.84 & 0.57 \\
\hline
\end{tabular}

The threat factor of the target changes randomly with the maneuvering of both weapon and target, and the simulation data obeys the uniform distribution of $(0.15,0.95)$.

Table3. Target Threat Factors

\begin{tabular}{llllll}
\hline Target & $\mathrm{M}_{1}$ & $\mathrm{M}_{2}$ & $\mathrm{M}_{3}$ & $\mathrm{M}_{4}$ & $\mathrm{M}_{5}$ \\
\hline $\begin{array}{l}\text { Threat } \\
\text { factor }\end{array}$ & 0.31 & 0.69 & 0.53 & 0.61 & 0.44 \\
\hline
\end{tabular}

The improved ant colony algorithm ${ }^{11}$ is used to allocate weapon targets, where the number of ants $\mathrm{A}=10$, the pheromone heuristic factor $\alpha=1.1$, the pheromone increment per unit length $\mathrm{M}=80$, and the pheromone volatilization coefficient $\quad \beta=0.7$. Use the two termination control strategies in 3.4 to control the running situation, and run the algorithm to get the results as shown in Figure 2.

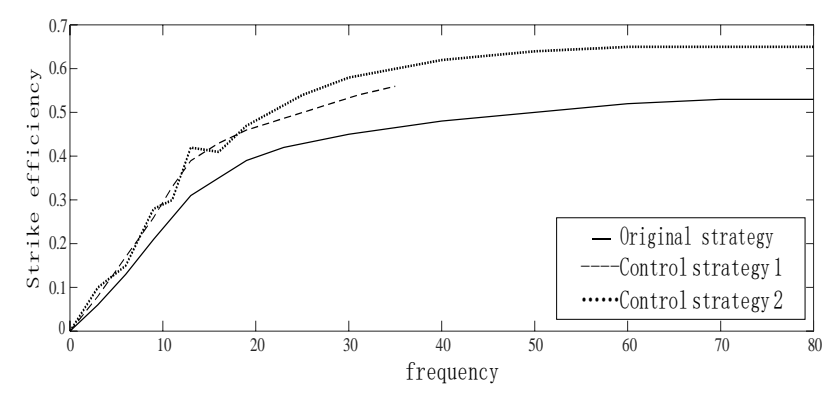

Fig2. Simulation renderings

It can be seen from the results in Figure 2 that, 
compared with the original model, the improved model can improve the attack efficiency and enhance the attack effect, and at the same time, two control strategies are adopted to monitor the operation situation ${ }^{12}$. Strategy 1 has more iterations, so its result is better than the output result of Strategy 2. When there is an urgent target after 35 times of calculation, the operation will be stopped immediately and the current optimal solution will be output. At this time, the output solution may not be the overall optimal solution, but it meets the goal of destroying the most urgent threat to our side in the shortest time, and conforms to the principle of dealing with urgent targets in synthetic detachment operations.

\section{CONCLUSION}

The battlefield application of synthetic detachment has its own characteristics. In view of these characteristics, this paper improves and optimizes the original weapon target allocation model, puts forward the concept of threat factor and adopts two termination control strategies to realize the emergency treatment of emergency targets. It is verified by an example that the concept of threat factor provides an effective method for dealing with emergency targets of synthetic detachment. The improved weapon target allocation model can effectively improve the attack efficiency of weapons against targets per unit time.

\section{References}

1. Liu Xu, Li Weimin, Song Wenjing. Target allocation of air defense operations considering overlapping launch areas [J]. Journal of Air Force Engineering University, 2013,14(6):30-33.

2. Fu ping-ping, Liu Zhao-ping. research on ant colony algorithm for optimal allocation of amphibious formation air defense firepower [J]. computer simulation, 2014,31(7):5-9.

3. Li Yongjun, Huang Zhuo, Guo Bo. A summary of weapon-target allocation [J]. Ordnance Automation, 2009,28(11):1-9.

4. Chang Tianqing, Chen Junwei, Hao Na, Ma Dianzhe. Ant Colony Algorithm Termination Control in Dynamic Weapon Target Allocation of Armored Units [J]. systems engineering and electronics, 2015,2,37(2):343-347.

5. Xu kehu, kong depeng, Chen Jinyu. cooperative decision model for dynamic weapon target allocation of synthetic detachment [J]. firepower and command and control, 2016,6(41):62-72.

6. KhoslaD.Hybridgenetic approachfor the dynamic weapon-target allocation problem[C]/Proc.of the SPIE,2001:248-263.

7. Zhang Jieyong, Yao Peiyang, Xin Wang, Zhou Xiangxiang. Multi-platform collaborative target allocation method based on time constraint [J]. systems engineering and electronics, 2011,6(25):1287-1292.

8. Chen Junwei, Chang Tianqing, Zhang lei, Yang
Guozhen. Two-stage WTA Model for Armored Unit Tactics Application [J]. systems engineering and electronics, 2016.38(6):1326-1327.

9. Zhao Jiankang, Zhu Yinggui, Xu Chunhe. Optimal fire distribution of tank company [J]. Fire and Command and Control, 2005,30(6):77-80.

10. Bai fan, Chang Tianqing, Wang qinzhao. WTA model of tank unit based on fuzzy fire fitness principle [J]. journal of system simulation, 2012,24(6):1161-1164.

11. Lee Z J,Lee W L.A hybrid search algorithm of ant colony optimization and genetic algorithm applied to weapon-target assignment problems[J].Lecture Notes IN Cumputer Science,2003,2690(1):278-285.

12. Chang Tianqing, Bai Fan, Li Yong, et al. Study on population initialization of intelligent optimization algorithm for solving WTA problem [J]. Computer Applied Research, 2013,30(5):1377-1380. 